\title{
CLINICOPATHOLOGIC CONFERENCE
}

\section{Young Male with Oro-genital Ulcers, Retroperitoneal Mass and Lymphadenopathy}

\author{
Hilal Bhat, M.D. ', Rafi Jan, M.D. ', Rais Malik, M.D. ${ }^{2}$, Munir Wani, M.D. ${ }^{3}$, Naseer Choh, M.D. ${ }^{4}$ \\ 'Department of Internal Medicine, ${ }^{2}$ Department of Pathology, ${ }^{3}$ Department of General Surgery, \\ ${ }^{4}$ Department of Radio diagnosis and Imaging SKIMS Srinagar
}

Dr Hilal Mohiuddin (Internal Medicine): 18 year old male student was admitted to this hospital with painful orogenital ulcers of mouth and severe odynophagia. He was in his usual state of health until approximately 25 days before admission when he developed diffuse ulceration of mouth with difficulty in swallowing and bleeding. He also noticed a painful non pruritic lesion on glans penis. He denied any constitutional symptoms. 20 days back he was admitted to another hospital.

On examination, he was found to have diffuse ulceration of buccal mucosa and palate, but no lymph node was enlarged. Spleen was moderately enlarged and there was a painful papule on glans penis. His blood counts, kidney and liver functions were normal and he was negative for HBsAg, HIV I \& HIV II. He was put on IV antibiotics, topical antifungals and oral antifungals. A USG abdomen on $4^{\text {th }}$ day of admission revealed splenomegaly and a mass in right iliac fossa. A CT abdomen performed after IV contrast revealed a large $(5.4 \times 4.7 \mathrm{~cm})$ enhancing well circumscribed mass displacing right psoas laterally \& iliac vessels anteriorly (Picture 1). Spleen was grossly enlarged. A neurosurgery opinion was sought and patient was discharged from the hospital. Six days before admission he was seen in neurosurgery clinic of our hospital and very next day in General surgery clinic. An exploratory laprotomy was planned.

\section{Correspondence}

Dr Hilal Mohi ud din Bhat, M.D.

Department of Internal Medicine SKIMS, Soura Srinagar email: hmohiudin@gmail.com

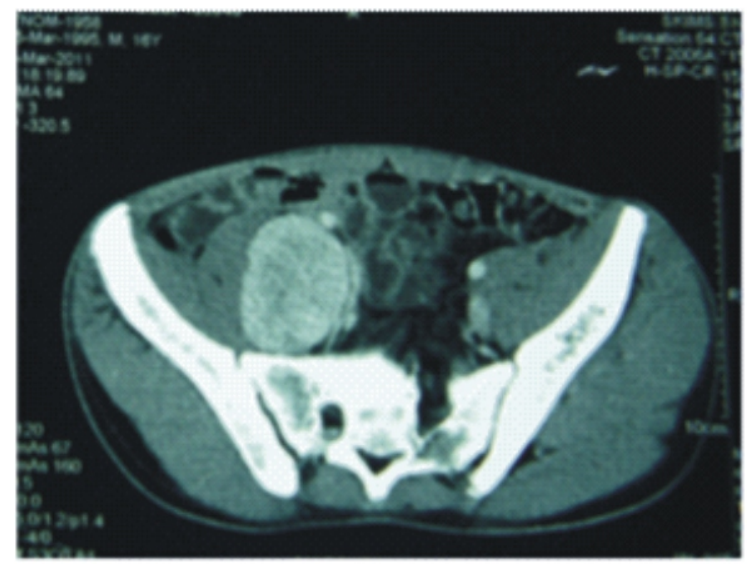

Picture 1

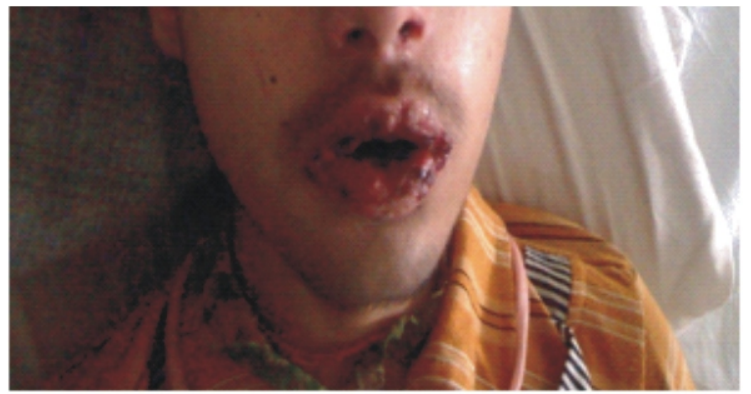

Picture 2

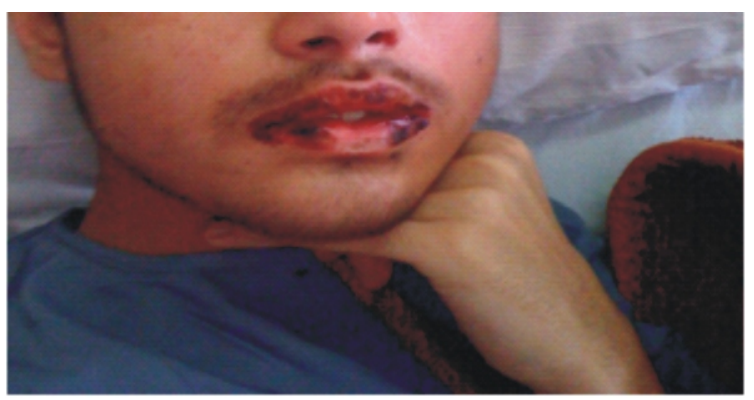

Picture 3 
He had no significant illness in the past. He was an unmarried student and had never smoked or used any illicit drugs. He did not travel outside. He lived with his parents. They were 5 siblings and were all healthy. Medication included topical and oral anti-fungals.

On examination, he appeared anxious. Temperature was $98^{\circ} \mathrm{F}$, the pulse 80 beats per minute, the blood pressure $110 / 70 \mathrm{~mm}$ of $\mathrm{Hg}$, the respiratory rate 18 per minute and oxygen saturation 98 percent while he was breathing ambient air. Abdomen was soft without tenderness or distention. There was a palpable mass deep in right iliac fossa. Spleen was soft and non tender and extended just above umbilicus. There was small tender peripheral lymphadenopathy in the cervical, submandibular and axillary areas. He had conjunctival injection and oral and pharyngeal painful erosions with haemorrhagic crusting of the lips (Picture 2). He had oral thrush. The reminder of examination was normal. Levels of serum glucose, electrolytes, phosphorus, magnesium, calcium and alkaline phosphatase and results of renal-function tests were normal. Tests for hepatitis B virus surface antibody and antigen and hepatitis $\mathrm{C}$ virus (HCV) antibody were negative. Serum lactate dehydrogenase \& uric acid was normal. An electrocardiogram was normal. Rests of the investigations are shown in table. He received topical antifungals and supportive care.

On fourth hospital day, oral swab showed insignificant bacterial growth. Blood culture was sterile and $\mathrm{KOH}$ mount of smear taken from mouth showed budding yeast cells. He continued to receive oral and topical anti fungals.

On fifth hospital day, fundus examination was normal, tzank smear was not suggestive, and pathergy test was negative. Antibiotic eye drops were added.

On seventh hospital day, he developed cough with thick mucoid sputum. Ulceration did not improve with any such measure. FNA cytology of cervical lymph node was reported as reactive. His oxygen saturation dropped transiently to $87 \%$ that again improved with oxygenation with a nasal prong. Hemodynamically he remained stable and chest roentgenogram was normal.

On tenth hospital day, bone marrow aspiration / biopsy was done and patient put on IV steroids, and there was some improvement in mucosal lesions (Picture 3 ). He continued to receive supportive treatment.

On twelfth hospital day, bone marrow was reported normal and patient was subjected to diagnostic procedure in the form of laprotomy.

\section{Complete Blood count}

\begin{tabular}{|c|c|c|c|c|c|c|c|c|}
\hline $\begin{array}{c}\text { Day of } \\
\text { hospital stay }\end{array}$ & $\mathbf{H b}$ & TLC & DLC & PLT & MCV & MCH & PCV & ESR \\
\hline Ist day & 11 & 5.11 & N60L35M5 & 168 & 87 & 26 & 41.8 & 21 \\
\hline $22-3-2011$ & 12.4 & 5.25 & N55L29 & 151 & 87.7 & 25.1 & 43.3 & 20 \\
\hline $24-3-2011$ & 11.9 & 4.44 & N52L29 & 158 & 88.2 & 25.4 & 41.3 & 22 \\
\hline $30-3-2011$ & 12.9 & 6.53 & N78L12M5 & 172 & 87 & 25.2 & 41.7 & 21 \\
\hline
\end{tabular}

\section{Biochemistry}

\begin{tabular}{|l|l|l|l|l|l|l|l|l|l|l|l|l|l|}
\hline Date & urea & Crt & BS & BIL & AST & ALT & ALP & TP & ALB & CA & CK & LDH & UA \\
\hline $4-3-2011$ & 20 & 0.59 & 88 & 0.43 & 36 & 33 & 156 & 7.58 & 4.22 & 9.2 & 78 & 112 & 5.1 \\
\hline $21-3-2011$ & 21 & 1.04 & 97 & 1.07 & 33 & 18 & 156 & 7.24 & 3.5 & 9.07 & 287 & 132 & 5.49 \\
\hline $30-3-2011$ & 25 & 0.52 & 128 & 1.37 & 26 & 8 & 283 & 6.18 & 3.02 & 9.1 & 82 & 142 & 4.5 \\
\hline $2-4-2011$ & 23 & 0.93 & 110 & 1.08 & 30 & 12 & 148 & 6.0 & 3.0 & 8.9 & 89 & 140 & 4.7 \\
\hline
\end{tabular}

\section{Arterial Blood Gases}

\begin{tabular}{|l|l|l|l|l|l|l|l|}
\hline Date & Na & K & PH & PO2 & SO2 & PCO2 & HCO3 \\
\hline $29-3-2011$ & 131 & 4.33 & 7.43 & 120 & 98.2 & 34 & 23 \\
\hline $5-4-2011$ & 129 & 3.98 & 7.47 & 100 & 98 & 34 & 23 \\
\hline $6-4-2011$ & 132 & 3.58 & 7.449 & 95 & 97 & 33 & 22.4 \\
\hline $8-4-2011$ & 140 & 3.41 & 7.365 & 100 & 97.4 & 38.4 & 21.5 \\
\hline
\end{tabular}

Coagulation profile

\begin{tabular}{|l|l|l|l|}
\hline Date & PT & INR & aPTT \\
\hline $31-3-2011$ & $14.1 / 11.0$ & 1.28 & $28.6 / 24$ \\
\hline
\end{tabular}




\section{DISCUSSION}

Dr Hilal Mohiuddin (Internal Medicine): The key features of this patient are a vascular retroperitoneal mass, generalized lymphadenopathy, massive splenomegaly and mucosal erosions.

Let me describe each key feature in the context of clinical summary:

\section{Retroperitoneal Mass}

The retroperitoneum is an underestimated site for benign and malignant neoplastic disease, and represents the second most common site of origin of primary malignant soft tissue tumors (sarcomas) after the deep tissues of the lower extremity ${ }^{1}$. The relative paucity of vital structures, and the abundance of loose connective tissue in this area, results in a generally late clinical presentation of space occupying lesions. Symptoms tend to be related to gastrointestinal, urinary or vascular compromise, when large lesional size and compression/invasion of adjacent structures severely limits the curative treatment options. Collectively, malignant tumours of the retroperitoneum are roughly four times more frequent than benign lesions, in sharp contrast to neoplastic disease occurring elsewhere in the body, where benign disease predominates. In adults the majority of retroperitoneal neoplasms are primary lymphoproliferative (Hodgkin's and non-Hodgkin's lymphoma) and parenchymatous epithelial tumours (renal, adrenal, pancreas), or represent metastatic disease from known or unknown primary sites elsewhere. Soft tissue (mesenchymal) tumours of the retroperitoneum are less common; nevertheless $15 \%$ of all primary sarcomas arise within the retroperitoneum, and consequently this represents the second most common site for the origin of malignant mesenchymal tumours, after the deep tissues of the lower extremity (thigh). It is the general experience that, although benign soft tissue lesions at all sites outnumber their malignant counterparts by a ratio of at least 100:1, in the retroperitoneum sarcomas are more prevalent than their benign counterparts. In comparison with soft tissue lesions elsewhere, however, the range of commonly encountered retroperitoneal mesenchymal neoplasms is more limited, with the most common lesions being of a lipomatous, smooth muscle or neural nature.
Retroperitoneal mass lesions

\begin{tabular}{|l|l|}
\hline Malignant & Benign \\
\hline $\begin{array}{l}\text { Well-differentiated } \\
\text { liposarcoma }\end{array}$ & Myolipoma \\
\hline $\begin{array}{l}\text { Dedifferentiated } \\
\text { liposarcoma }\end{array}$ & Neurofibroma \\
\hline $\begin{array}{l}\text { Leiomyosarcoma } \\
\text { sheatignant peripheral nerve }\end{array}$ & \\
\hline $\begin{array}{l}\text { Desmoplastic small round } \\
\text { cell tumour }\end{array}$ & \\
\hline $\begin{array}{l}\text { Pleomorphic sarcoma, } \\
\text { undifferentiated }\end{array}$ & \\
\hline $\begin{array}{l}\text { Kaposiform } \\
\text { haemangioendothelioma }\end{array}$ & \\
\hline
\end{tabular}

Schwannoma, including the cellular variant (cellular schwannoma), is the most common neurogenic soft tissue tumour occurring in the retroperitoneum. Clinically, these lesions have a broad age range of presentation, and often occur paravertebrally.

MPNSTs are defined as malignant mesenchymal tumours arising from, or differentiating towards, cells of the peripheral nerve sheath, but excluding epineurial tumours and tumours arising from the neural vasculature. Roughly two-thirds of cases are associated with a preexisting neurofibroma, while approximately half of MPNSTs arise in patients with neurofibromatosis I (NF I) (presumably having arisen within a neurofibroma). MPNSTs are tumours of adults, although the age at presentation in patients with NF I, is younger than in unaffected patients. MPNST is a high-grade tumour with an extremely poor prognosis $(20 \pm$ $30 \%$ 5-year survival).

Malignant retroperitoneal paragangliomas range from $30 \%$ to $50 \%$. Paragangliomas metastasize approximately in $20 \%$ to $42 \%$ of the cases. Dissemination can be hematogenous or through the lymphatic system, with the most common site of metastasis being the regional lymph nodes, bone, lung, and liver. Because benign and malignant paragangliomas have the same histological appearance, the best predictor for outcome is metastasis or recurrence. Functional paragangliomas secrete norepinephrine and normetanephrine and account for $30-60 \%$ of the tumors. If a secretory tumor is present, the patients undergo paroxysmal episodic hypertension, as well as the typical triad of symptoms associated with pheochromocytomas: palpitations, headache, and profuse sweating. The 
nonsecretory type most commonly presents as abdominal pain or mass; a large proportion of these tumors are incidentally discovered in normotensive patients during imaging evaluation for other reasons. The diagnosis is usually established with high urine catecholamine metabolites, VMA, and metanephrine levels. They are commonly located in the para-aortic region, and they may be confused with other retroperitoneal tumors, especially pancreatic tumors.

Lymphoproliferative disorders like non-Hodgkins and Hodgkins lymphoma make a strong possibility in this patient in view of lymphadenopathy and splenomegaly. But a normal hemogram and bone marrow examination in addition to reactive lymph node cytology do not strongly support any of these. Castleman's tumors a neoplasms of lymphatic origin, also known as giant lymph node hyperplasia or benign giant lymphoma and IgG4-related disease characterized by mass-forming lesions in mainly exocrine tissue due to lymphoplasmacytic infiltrates and sclerosis, increased serum IgG4 level, and IgG4-positive plasma cells in the affected tissues are also a possibility .

\section{Role of fine needle aspiration/needle core biopsy in retroperitoneal tumors}

Depending on the site of the retroperitoneal lesion, fine needle aspiration/needle core biopsy may or may not be practically tenable. Fine needle aspiration (FNA), when possible, may be very useful as a first step in resolving the differential diagnosis of primary or metastatic carcinoma, a lymphoproliferative disorder, or a mesenchymal tumor ${ }^{2}$. Once carcinoma and lymphoma have been excluded, its usefulness is limited since attempts at classification of mesenchymal tumours on FNA material, except in expert hands, is probably unreliable and not advisable. The role of a needle core biopsy is probably controversial. While most retroperitoneal soft tissue lesions are probably malignant (except schwannoma), and will need some form of definitive operative procedure (including schwannomas as a result of a local mass effect), the risk of tumour spill following a needle core biopsy needs to be weighed up against the possibility/need for an optimal surgical intervention $^{3}$.

\section{Role of radiography in retroperitoneal tumors}

Among neurogenic tumors, schwannomas are the most common tumor of peripheral nerves. Schwannomas are well encapsulated and contain cells that are identical to
Schwann cells. Their MR imaging appearance depends on the types of tissue they contain. Myxoid tissue is hyperintense on T2-weighted images, cellular tissue is hypointense on both T1- and T2- weighted images, and solid fibrous tissue enhances on contrast-enhanced images. Neurofibromas tend to have high signal intensity on T2weighted MR images and are often multiple and associated with neurofibromatosis. Ganglioneuromas are typically located along the sympathetic chain and tend to be larger, more rounded, and contain calcification more frequently than nerve sheath tumors. The relatively younger age of affected patients may aid in differentiating ganglioneuromas from other neurogenic tumors. Extremely hypervascular tumors such as paragangliomas sometimes contain hemorrhagic necrosis and manifest with fluid-fluid levels. Extraadrenal paragangliomas are more frequently malignant (up to $40 \%$ of cases) than adrenal pheochromocytomas. Lymphomas are the most commonly encountered tumors composed of small round cells. They are homogeneous, with minimal contrast enhancement at $\mathrm{CT}$ and relatively low signal intensity at T2-weighted MR imaging. An exception is primitive neuroectodermal tumor (PNET), which often appears heterogeneous at MR imaging. The imaging manifestations of Castleman's disease are closely related to the pathological characteristics of the lesions. Most lesions appear as nonspecific, welldefined homogeneous masses on sonography and CT scans. Konno et al described the disease as characterized by a large feeding artery penetrating the nodal hilum and prominent arteries in the periphery on a Doppler sonogram. Several cases reported showed that the Castleman's disease enhancement mode was similar to that of large arteries on contrast enhanced CT scans. High enhancement manifestations can be attributed first to the abundance of blood vessels in the HV type, and also focal vascular proliferation accompanied by abnormal proliferation and dilation of capillary vessels ${ }^{4}$.

\section{LYMPHADENOPATHY}

In the present clinical picture generalized lymphadenopathy is consistent with a lymphoproliferative disorder or multicentric castleman's disease. Evaluation of lymphadenopathy begins with FNAC. The fine needle aspiration (FNA) cytologic diagnosis of non-Hodgkin's lymphoma (NHL) depends upon finding a relatively monotonous population of lymphoid cells in smears. The success rate of FNA cytology ranges from $80 \%-90 \%$ in 
diagnosis of NHL and from $67.5 \%-86 \%$ in its subtyping. The cytodiagnosis of Hodgkin's disease (HD) depends upon demonstration of Reed-Sternberg cells or Hodgkin's cells amongst appropriate reactive cell components. The diagnostic accuracy of FNA cytology for HD has also been invariably high $(>85 \%)$. The differential diagnostic problems for NHL include a group of small round cell tumors, nonlymphoid acute leukemias and HD. FNA cytology remains the first line of investigations (screening test) used in cases of lymphadenopathy. Besides initial diagnosis of lymphoma, it helps in detection of residual disease, recurrences and progression of low-grade to highgrade lymphoma, and helps in staging the disease. The FNA of cervical lymph node was reported reactive. Reservations have been expressed regarding the efficacy of cytology in separating florid reactive hyperplasia from low-grade malignant lymphoma. It has been found that the overall diagnostic results improved after repeating the FNAC and repeating FNAC is useful and should be considered under some circumstances, especially in the case of nondiagnostic cervical lymph node aspirates ${ }^{5}$.

\section{SPLENOMEGALY}

Although the differential diagnosis for splenomegaly is broad, entities that cause massive splenomegaly are limited ${ }^{6}$.

\begin{tabular}{|l|l|}
\hline $\begin{array}{l}\text { Causes Of Massive Splenomegaly (> 8cm Below } \\
\text { Costal Margin Or Wt }>\text { 1kg) }\end{array}$ \\
\hline Cml & Cll \\
\hline Lymphomas & Sarcoidosis \\
\hline Hairy Cell Leukemia & Aiha \\
\hline Myelofibrosis & Thalassemia Major \\
\hline Polycythemia Vera & Visceral Leishmaniasis \\
\hline Gaucher's Disease & $\begin{array}{l}\text { Hyperrective Malarial } \\
\text { Splenomegaly Syndrome }\end{array}$ \\
\hline MAC & $\begin{array}{l}\text { Diffuse Splenic } \\
\text { Hemangiomatosis }\end{array}$ \\
\hline
\end{tabular}

Keeping in mind the patient presentation and investigations there are no definite causes for his massive splenomegaly. Many lymphomas may involve the spleen and present with splenomegaly. Diffuse large-B-cell lymphoma is the most common lymphoma in adults in the United States. But FNA cytology and bone marrow examination was not suggestive of any lymphoma.

\section{Muco-cutanous lesions}

In the light of patient overall presentation, this kind of muco-cutaneous ulceration can take us to a specific diagnosis and the ultimate cause of death.

Behçet's syndrome is a multisystem disorder presenting with recurrent oral and genital ulcerations as well as ocular involvement. The diagnosis is clinical and based on internationally agreed diagnostic criteria

\section{Diagnostic Criteria of Behçet's Disease}

Recurrent oral ulceration plus two of the following:

Recurrent genital ulceration

Eye lesions

Skin lesions

Pathergy test

The syndrome affects young males and females from the Mediterranean region, the Middle East, and the Far East. Males and females are affected equally, but males often have more severe disease. The ulcers are usually painful, are shallow or deep with a central yellowish necrotic base, appear singly or in crops, and are located anywhere in the oral cavity. The ulcers persist for 12 weeks and subside without leaving scars. The genital ulcers are less common but more specific, do not affect the glans penis or urethra, and produce scrotal scars. None of such features were seen in our patient. Skin involvement includes folliculitis, erythema nodosum, an acne-like exanthem, and infrequently, vasculitis. Nonspecific skin inflammatory reactivity to any scratches or intradermal saline injection (pathergy test) is a common and specific manifestation that was negative in him. Eye involvement with scarring and bilateral panuveitis is the most dreaded complication, since it occasionally progresses rapidly to blindness ${ }^{7}$. In this patient conjunctival congestion was clearly separable from an absent uvietis. Furthermore there were no other systemic symptoms specific to this disorder.

\section{Paraneoplastic pimphigus}

Paraneoplastic pemphigus (PNP) is a life threatening autoimmune blistering disease, which is commonly associated with lymphoproliferative neoplasms. It is characterized by painful mucosal erosions with a polymorphous skin eruption (not always defined by 
bullae) in association with an occult or verified neoplasm. Lymphoproliferative neoplasms comprise the majority of neoplasms associated with PNP. The most frequently documented is non- Hodgkin lymphoma (38.6\%), followed by chronic lymphocytic leukemia (18.4\%) and Castleman's disease (18.4\%). Rarely, nonhematologic neoplasms are involved in PNP and include epithelial-origin carcinomas (pancreas, colon, breast prostate, squamous cell carcinoma, basal cell carcimona, and bronchogenic carcinomas) (8.6\%) and mesenchymal-origin sarcomas (reticulum cell sarcomas, liposarcomas, leiomyosarcomas, and dendritic cell sarcomas)(6.2\%) Mortality due to the disease has been reported to be as high as $90 \%$. There has also been well-documented evidence of involvement of internal organs, such as lungs, thyroid, kidney, smooth muscle, and gastrointestinal tract. Such internal organ involvement has led to the proposal that the term, paraneoplastic pemphigus, should be replaced by the term, paraneoplastic autoimmune multiorgan syndrome (PAMS), to more accurately describe the full spectrum of presentations and pathologic findings associated with this condition, although this proposed change has been met with mixed reaction by professionals in the field. The clinical presentation of PNP, although highly varied, typically begins with a florid, painful eruption of mucous membrane lesions, which may involve the oropharynx, nasopharynx, tongue, vermillion of the lips, conjunctiva, anogenital region, or esophagus. The onset of cutaneous lesions has a variable delay after the appearance of mucous membrane lesions, ranging from days to months. Common causes of mortality in PNP include overwhelming sepsis and complications due to treatment for the underlying neoplasm. A more common cause of mortality in children and those where the underlying malignant condition in Castleman's disease is bronchiolitis obliterans. This condition is characterized by the sloughing of the epithelial lining of the bronchioles, which can lead to complete obstructions of terminal bronchioles and individual alveoli. This aspect of the disease is the only commonly reported internal manifestation of PNP/PAMS, and although other internal organs have reported autoantibody reactivity, no clinical manifestations or complications have been directly attributed to the disease process. PNP can mimic a variety of dermatological disease including pemphigus vulgaris (Superficial vesicles with occasional erythema), erythema multiforme (Scaling erythematous papules with occasional ulceration or erosions), erosive lichen planus (Violaceus papules) and acute SLE (systemic features). So, the correct diagonosis is always delayed. Diagonosis is by histology and immunoflorecense both of which are not available in our case. But, Castleman's tumor should be always suspected when a retroperitoneal mass is combined with PNP. CD has been found to be associated with various cutaneous changes, including erosive mucocutaneous lesions, erosive lichen planus and lichen planus type lesions.

\section{IgG4-related disease}

Recently recognized syndrome characterized by mass-forming lesions due to lymphoplasmacytic infiltrates and sclerosis, increased serum IgG4 level, and IgG4-positive plasma cells in the affected tissues. Recent studies have reported that IgG4-related disease sometimes involves the systemic lymph nodes but clinicopathological characteristics have not been well documented yet. IgG4-related disease sometimes involves regional and/or systemic lymph nodes, and is often clinically and/or histologically suspected to be multicentric Castleman's disease and/or malignant lymphoma ${ }^{9}$ Interleukin-6 and C-reactive protein are important as differential diagnostic markers between systemic IgG4-related lymphadenopathy and multicentric Castleman's disease. Masaki et al have reported that IgG4-related disease is not associated with an elevated serum interleukin- 6 level, and cited measurement of serum interleukin- 6 as an important 
tool of differential diagnosis.

Dr Rafi Jan (internal medicine): After listening to this descriptive background this patient could have a Para neoplastic syndrome manifesting as pemphigus but skin biopsy and IF should have cleared the doubt. He didn't fit in criterion for behcet's disease although an IgG4 disease couldn't be rulled out without the biopsy of lesion. Paraganglioms can be non-secreatory at times but associated splenomegaly again takes us to a lymph reticular disease either a benign or malignant one.

Dr Seema Qayoom (Dermatology \& Venerology): Painful blistering mucosal lesions point towards pemphigus and since lymphoproliferative disorders are the major causes of paraneoplastic pemphigus, I am of the same openion and the abdominal mass described above with splenomegaly do support my claim.

Dr Muneer wani (General Surgery): It was a highly vascular tumor capsulated and large. Although we had no difficulties in achieving hemostasis, we had kept all possible outcomes and were in advance ready for any complication.

Dr Samoon Jeelani (Clinical Hematology): I had the opportunity to review the bone marrow aspiration and biopsy slides. There were no abnormal bone marrow features including any plasma cells or blasts. With an abdominal mass and a big spleen I was expecting something in BM but that doesn't rule out a localized/limited lymphoma or castleman's disease. Biopsy was unremarkable.

Dr Rais (Pathology): We received biopsy of this patient labeled as retroperitoneal mass. Grossly there were multiple tissue pieces, largest of piece was globular well circumscribed and well encapsulated mass measuring $4 \times 6 \mathrm{~cm}$. Cut section was grey white soft homogenous with fibrotic areas. Out of rest tissue pieces 7 lymph nodes were dissected out.

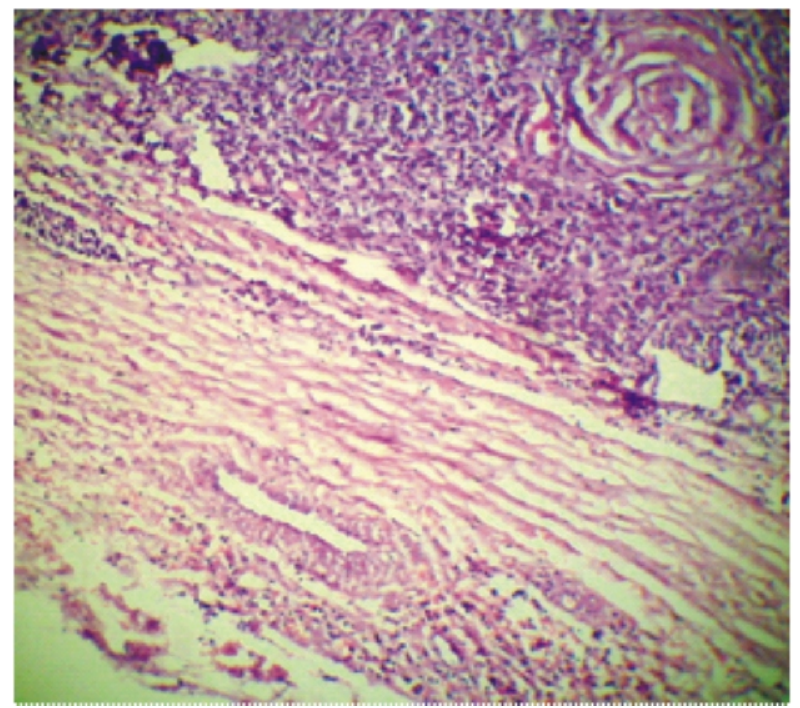

Picture 4

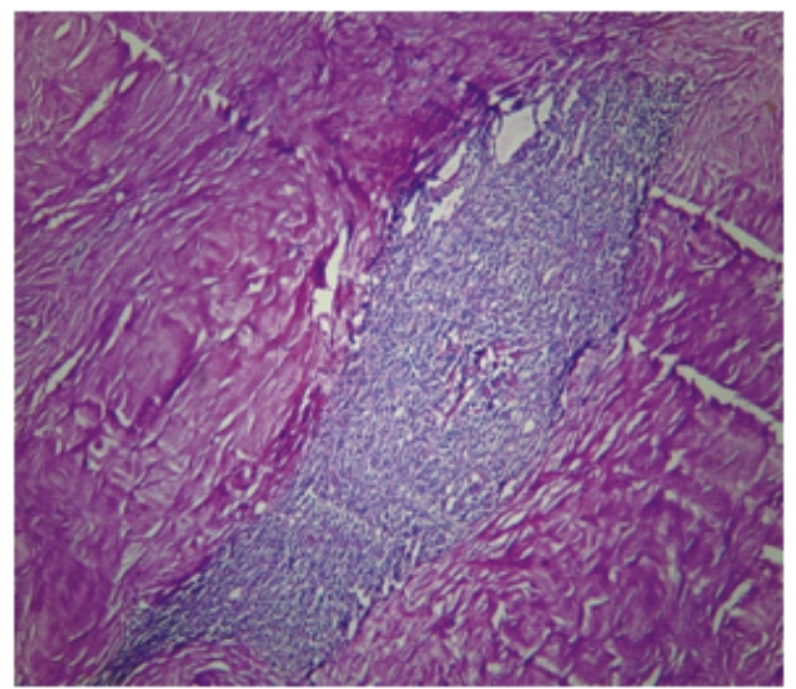

Picture 5

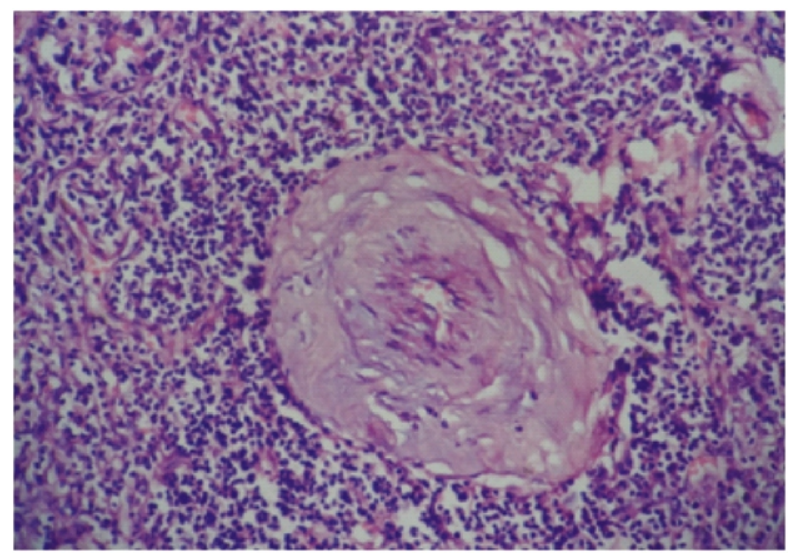

Picture 6 
Microscopically (Figure 4, 5 \& 6) thickened capsule of the mass was noted; normal architecture of lymph node was lost. Cortex as well as medulla showed follicle formation of varying size. Centre of the follicle showed vessel with onion peel hyalinization of vessels. Thick hyalinized bands were also noted traversing the tissue. Rest of all the nodes showed reactive lymphadenitis. We also received bone marrow biopsy. which showed unremarkable marrow. In our differential diagnosis many things came in. yes, Hodgkins lymphoma can have such changes, but such changes are focal, not many eosinophils or plasma cells were identified. Above all no R-S cell was identified. Non hodgkins lymphoma of follicular lymphoma variant can have these changes, but again focally seen, also patient is of young age, wall is thin and nowell defined follicles. But the features which are noted microscopically fits most in hyaline vascular type of castleman's lymphadenopthy, main thing which goes againt diagnosis is that this variant is most of times multicentric, but unicentric type can also be seen rarely, So our diagnosis is uni-centeric castle man's lymphadenopathy (hyaline vascular type).

Final Diagnosis: Unicentric Castleman's disease.

\section{Management (Dr Hilal Mohiuddin):}

Castleman disease (CD), also known as angiofollicular lymph node hyperplasia, was first reported in 1954 by Benjamin Castleman. He described a 40-year-old male with a mediastinal mass characterized histologically by lymph node hyperplasia and follicles with small, hyalinized foci ${ }^{10}$. Present understanding is that $\mathrm{CD}$ as a heterogeneous cluster of disorders, with distinct unicentric CD (UCD) and multicentric CD (MCD) subtypes, with identified fundamental roles of human herpesvirus- 8 (HHV-8) and interleukin-6 (IL-6) in a significant proportion of cases. Complete surgical resection is curative for UCD, leading to excellent long-term outcomes with 10-year overall survival rates in excess of $95 \%$. If this is not feasible, the optimal treatment strategy is not well-defined. Debulking surgery should be considered, particularly if there are local symptoms at presentation or if there is a risk of compressing nearby critical structures ${ }^{11}$.

For multi centric $C D$ various systemic therapies have been utilized in MCD, including cytotoxic chemotherapy, antibodies directed against CD20 as well as IL-6 and its receptor, immunomodulators, bortezomib, and antiviral agents. While they all appear to have disease activity, the literature documenting their use is mainly confined to case reports or small series of patients ${ }^{12}$.

The advent of monoclonal antibody therapy targeting the IL-6 pathway has significantly impacted the treatment of patients with idiopathic MCD. Agents that have been studied include siltuximab, a chimeric monoclonal antibody to IL-6, and tocilizumab, a recombinant humanized monoclonal antibody that blocks the IL-6 receptor.

\section{REFERENCES}

1. J. Frans Graadt Van Roggen \& Pancras C.W. Hogendoorn; Soft tissue tumours of the retroperitoneum: Sarcoma (2000) 4, 17-26.

2. Hopper KD: Percutaneous, radiographically guided biopsy: a history. Radiology 1995;196:329-333

3. Yuki Tomozawa, Yoshitaka Inaba, Hidekazu Yamaura, Yozo Sato, Mina Kato, Takaaki Kanamoto, Makoto Sakane; Clinical Value of CT-Guided Needle Biopsy for Retroperitoneal Lesions: Korean J Radiol 2011;12(3):351-357.

4. Mizuki Nishino, Katsumi Hayakawa, Manabu Minami, Akira Yamamoto, Hiroyuki Ueda, Kosho Takasu Primary Retroperitoneal Neoplasms: CT and MR Imaging Findings with Anatomic and Pathologic Diagnostic Clues: Radio Graphics 2003; 23:4557.

5. Wilkinson AR, Mahore SD, Maimoon SA. FNAC in the diagnosis of lymph node malignancies: A simple and sensitive tool. Indian Journal of Medical and Paediatric Oncology: Official Journal of Indian Society of Medical \& Paediatric Oncology. 2012;33(1):21-24. doi:10.4103/0971-5851.96964.

6. Suzuki Kurokawa M, Suzuki N. Behcet's disease: Clin Exp Med. 2004 Sep;4(1):10-20. Review.

7. D.Mimouni, G.J. Anhalt, Z. Lazarova, S. Aho, S. Kazerounian, D.J. Kouba, J.M. Mascaro And H.C. Nousari: British Journal of Dermatology 2002, 147, 725-732;

8. Yasuharu Sato, Masaru Kojima, Katsuyoshi Takata, Toshiaki Morito1, Hideki Asaoku, Tamotsu Takeuchi, Kohichi Mizobuchi, Megumu Fujihara, Kazuya Kuraoka, Tokiko Nakai, Kouichi Ichimura, Takehiro Tanaka, Maiko Tamura, Yuriko Nishikawa and Tadashi 
Yoshino; Systemic IgG4-related lymphadenopathy: a clinical and pathologic comparison to multicentric Castleman's disease; Modern Pathology (2009) 22, 589-599.

9. Case records of the Massachusetts general hospital weekly clinicopathological exercises: case 40011. N
Engl J Med. 1954;250(1):26-30.

10. Talat N, Belgaumkar AP, Schulte KM. Surgery in Castleman's disease: a systematic review of 404 published cases. Ann Surg. 2012;255(4):677-684. 